\title{
Aproximación a la competencia digital docente en la formación profesional
}

\section{Approach to digital teaching competence in vocational training}

\author{
Cristóbal Suárez-Guerrero \\ Universitat de València. València, España \\ cristobal.suarez@uv.es \\ Alicia Ros-Garrido \\ Universitat de València. València, España \\ alicia.ros@uv.es \\ Jorge Lizandra \\ Universitat de València. València, España \\ jorge.lizandra@uv.es
}

\begin{abstract}
Resumen
El mundo requiere personas con conocimientos, habilidades y actitudes que permitan un desarrollo completo de la ciudadanía en un ecosistema digital. Una respuesta educativa a esta exigencia social es la competencia digital. Así, esta finalidad exige niveles óptimos de desarrollo de la Competencia Digital Docente (CDD), en especial en el sector de la Formación Profesional (FP). Con la idea de atender este contexto educativo, en este trabajo se propone un marco de comprensión que integra de forma unitaria la visión sobre la pedagogía, los recursos y la evaluación para entender, evaluar y proponer mejoras en la competencia digital del profesorado de FP. Empleando un enfoque cualitativo, se realizaron once entrevistas en profundidad sobre la CDD del profesorado de FP de siete centros educativos en la provincia de Valencia (España). Estos datos fueron analizados desde la caracterización de cuatro escenarios educativos con tecnología (aprendizaje individual, la enseñanza con TIC, aprendizaje colaborativo y autoaprendizaje del alumnado). La principal conclusión detectada es que los docentes entrevistados tienen conocimientos de algunos recursos y aplicaciones tecnológicas, actitud y capacidad para incorporarlos a su práctica docente. En cambio, no integran la tecnología en la evaluación de los aprendizajes al mismo nivel competencial que en las otras dimensiones.
\end{abstract}

Palabras clave: Competencia digital, docente, formación profesional, evaluación, recursos y pedagogía.

\begin{abstract}
World requires people with knowledge, skills and attitudes that allow a full development of citizenship in a digital ecosystem. An educational response to this social demand is digital competence. Thus, this purpose requires optimal levels of Digital Teaching Competence (DTC), especially in the Vocational Education Training (VET) sector. With the aim of attending to this educational context, this work proposes a understanding framework that integrates the vision of pedagogy, resources and evaluation to understand, evaluate and propose improvements in the digital competence of VET teachers. Using a qualitative approach, eleven in-depth interviews were conducted on the DTC of VET teachers from seven schools in the province of Valencia (Spain). These data were analysed from the categorization of four educational scenarios with technology (individual learning, teaching with ICT, collaborative learning and student self-learning). The main conclusion detected is that interviewed teachers have knowledge of some technological resources and applications, attitude and ability to incorporate them into their teaching practice.
\end{abstract}


However, they do not integrate technology in the learning assessment at the same competence level as in the other dimensions.

Key words: Digital competence, teacher, vocational training, assessment, pedagogy and resources.

\section{Introducción}

La competencia digital se ha convertido en una dimensión educativa de la ciudadanía y en una necesidad formativa profesional de primer orden en la sociedad actual (Ananiadou y Claro, 2009). Hoy, ser competente digital es una finalidad educativa clave y transversal para muchos niveles y perfiles profesionales (European Commission, 2010) ya que su desarrollo tiene un gran en impacto en el campo laboral (World Economic Forum, 2018). Estos grandes, acelerados e inquietantes cambios tecnológicos que la sociedad está experimentando (Saran, Chapman y Sharma, 2018) requieren una respuesta educativa que permita a las personas desarrollar tanto conocimientos, habilidades y actitudes para el mundo digital (Cobo, 2019; Loveless y Williamson, 2013).

Uno de los aspectos clave que tiene el desarrollo de la competencia digital es que ha permitido superar la visión técnica del uso de la tecnología por una más amplia. Este es el caso de la idea muy extendida sobre el Nativo Digital (Prensky, 2001). En muchos sectores de la sociedad se afianzó la idea que de que para ser eficiente con la tecnología digital era suficiente haber nacido en un entorno digital. Esta idea ya no es aceptada por su falta de rigor empírico (Kirschner y De Bruyckere, 2017).

¿Si ser competente digital no tiene que ver con la edad, entonces qué implica? Un primer foco de atención es entender que, lejos de la pertenencia o no a una generación tecnológica aparentemente solvente, lo esencial es ver cómo las personas construyen sus identidades digitales, ya sea como residentes o visitantes del entorno tecnológico (White y Le Cornu, 2011). También es posible entender, sin desestimar ciertas habilidades sociales y lúdicas de los jóvenes al usar la tecnología, que para ser competente digital hace falta habilidades asociadas a la creación y transferencia del conocimiento (Valtonen et al, 2011). Además, como usuario de la red, es cada vez más importante no solo tener conciencia de dónde se accede y qué es lo que se comparte en la red, sino también lo que los algoritmos tecnológicos gestionan nuestra conducta (Szymielewicz, 2019).

Ya que la noción de competencia digital aborda otras dimensiones más allá de la relación persona-medios de comunicación, se ha buscado ganar precisión en su concepto y se ha avanzado en la identificación de las áreas que requieren un desarrollo educativo. Es por ello que en el panorama educativo, además de los ya clásicos temas en educación y tecnología, como los estudios sobre el impacto de las TIC en la educación y el aprendizaje, el diseño instruccional con tecnología, el aprendizaje basado en tecnología, el aprendizaje asistido por tecnología o el e-learning (Badia, 2015), ha surgido una línea de investigación y desarrollo educativo que busca conocer, impulsar y evaluar los conocimientos, actitudes y habilidades de las personas para usar la tecnología con solvencia (Goodfellow y Lea, 2016).

Aproximación a la competencia digital docente en la formación profesional. Suárez-Guerrero, Ros-Garrido y Lizandra. 


\subsection{Competencia Digital Docente (CDD)}

La necesidad de formación del docente en torno a la tecnología, en la que cabe la competencia digital como concepto específico, es vista como la segunda necesidad formativa docente más importante a nivel mundial (OCDE, 2019). Además del impulso de la competencia ciudadana como expectativa formativa en el ámbito de la educación básica, la competencia digital se está consolidando como una dimensión clave de la función y actividad docente. Hoy, el desarrollo de la noción de Competencia Digital Docente (CDD) forma parte de una manera de ser, pensar y actuar del docente (Fainholc, Nervi, Romero \& Halal, 2015; Gisbert, Esteve y Lázaro, 2019). Pero, aunque la CDD aluda a aspectos tecnológicos, como es lo digital, debe quedar claro que hablar de esta competencia en el docente implica una forma de representación pedagógica del hecho digital (Suárez-Guerrero, 2014). La CDD es un concepto pedagógico en toda regla.

Ahora bien, si bien es cierto que la competencia digital forma parte de las competencias necesarias para el aprendizaje permanente de cualquier profesional (Ala-Mutka, Punie y Redecker, 2008), la CDD se ha convertido en parte indispensable de la formación y la actividad del profesorado en la actualidad (Esteve, 2015; Salinas, de Benito y Lizana, 2014). Es decir, la competencia digital es una de las competencias clave de la función docente hoy en día (Hatlevik, 2017; Gudmundsdottir y Hatlevic, 2018).

Para graficar el panorama se puede señalar que los intentos por entender y caracterizar la $\mathrm{CDD}$, en algunos casos recibiendo denominaciones diferentes, no son homogéneos. A pesar de sus matices, estos modelos tienen dos características fundamentales: ofrecen indicadores o niveles que describen cómo es esa competencia en el docente y todos tienen la clara orientación de superar la visión técnica de esta competencia. Entre los modelos de CDD más destacados se puede señalar:

- "Modelo NETS-T" (ISTE, 2008), que incluye cuatro niveles (Inicial, medio, experto y transformador), asume los siguientes criterios: aprendizaje y creatividad de los estudiantes, experiencia de aprendizaje y evaluación, trabajo y aprendizaje en la era digital, ciudadanía digital y responsabilidad y desarrollo profesional y el liderazgo.

- "UNESCO ICT Competency Framework for Teachers" (UNESCO, 2008, 2011), que asume tres niveles (adquisición de nociones básicas, profundización del conocimiento y generación de conocimiento), toma en cuenta criterios como: currículum y evaluación, pedagogía, TIC, organización y administración y aprendizaje profesional del Profesor.

- "Marco Común de Competencia Digital Docente. V 2.0” (MECD, 2013) elaborado por el INTEF sobre la base de la propuesta DigComp de la Unión Europea, asume tres niveles (básico, intermedio y avanzado) y toma en cuenta cinco áreas, la mismas que la competencia general: información, comunicación, creación de contenido, seguridad y resolución de problemas.

- "DigCompEdu", que nace asociado a "The Digital Competence Framework for Citizens" (Carretero, Vuorikari y Punie, 2017; Vuorikari, Punie, Carretero y Van den Brande, 2016; Ferrari, 2013), propone 6 niveles: básicas, newcomer

Aproximación a la competencia digital docente en la formación profesional. Suárez-Guerrero, Ros-Garrido y Lizandra. 
(A1) y explorer (A2), intermedias, integrator (B1) y expert (B2) y altos de desarrollo competencial, leader (C1) y pioneer (C2) (Redecker, 2017).

Todos estos trabajos forman parte de una evolución del concepto de competencia digital docente. Aunque se trata de un concepto en torno a constantes pedagógicas, es importante ver que la variable tecnológica imprime un sesgo y una velocidad diferentes a otros conceptos educativos (Williamson, 2018). En este trabajo se opta partir del enfoque "DigCompEdu, por su actualidad y por su pertinencia para el contexto de la formación profesional.

\subsection{Contexto del profesorado de formación profesional y tecnología}

El ámbito académico ha atendido escasamente al contexto de la Formación Profesional (FP) (OCDE, 2011). El campo de la didáctica en España ha ignorado en gran medida la FP (Marhuenda-Fluixá y Ros-Garrido, 2015). Dichas tendencias deben cambiar puesto que la FP es una herramienta de cambio y mejora social (Marhuenda-Fluixá, 2012) que puede considerarse como una opción formativa y laboral real y de futuro cada vez más importante (Cedefop, 2020).

Un primer acercamiento a la tecnología desde la perspectiva de la FP nos lleva a una conclusión clara y sencilla: en la medida en que la FP está relacionada con el mercado laboral y el sistema productivo, el uso de tecnologías en constante cambio a través de la digitalización y la automatización de los procesos tendrá un impacto al menos en dos ámbitos, la actualización del plan de estudios para tener en cuenta esos cambios, por un lado, así como el uso de las máquinas y los recursos que se utilizan en la producción para los procesos de enseñanza también, ya sea en las instalaciones de las empresas o también transferidos a las escuelas donde se estudia la FP, ya sea en las clases o en el taller. Obviamente, hay diferencias entre sectores profesionales, pero el proceso de actualización del curriculum prescrito establecido en el Catálogo Nacional de Cualificaciones Profesionales (CNCP) es muy lento. Así, dicha actualización pasa al curriculum moldeado por el profesorado y puede realizarse con la implicación directa del contexto real de trabajo: las empresas (dónde el estudiantado realiza las prácticas u otras que el profesorado detecte como claves en el sector profesional correspondiente). En este proceso el estudiantado también podría contribuir ya que, en los últimos años, se ha producido un cambio de su perfil (parte del estudiantado son personas adultas que retornan a la formación profesional), ya sea por su propia competencia digital (la consideración de nativos digitales, atribuida erróneamente al alumnado más joven), como por su previa experiencia profesional en el sector o, en general, en su carrera profesional.

Respecto al uso de la tecnología en educación, el estudiantado de FP señala las siguientes ventajas (Salas-Ruiz y Sánchez-Rivas, 2019): comunicación en tiempo real, superación de barreras geográficas, distribución y entrega de tareas desde cualquier contexto (no solo el aula-clase), desarrollo de otras capacidades que no se pueden hacer "a mano", y la posibilidad de tener clases más dinámicas y amenas. También otra investigación con estudiantes del sector electromecánico considera las tecnologías como elemento motivador en el aprendizaje y esenciales en su futuro laboral (Fabregat y Gallardo, 2019). Aunque no todo el profesorado está preparado para utilizar y desarrollar su competencia digital (Fabregat y Gallardo, 2019), también es bueno señalar que el estudiantado FP

Aproximación a la competencia digital docente en la formación profesional. Suárez-Guerrero, Ros-Garrido y Lizandra. 
también tiene una presencia importante de personas adultas, y muchos preferirían el uso tradicional de papel, pizarra y bolígrafo (Salas-Ruiz y Sánchez-Rivas, 2019). En general, el uso de las nuevas tecnologías se considera un avance y ayuda en el proceso de enseñanza y aprendizaje, y también para el estudiantado de formación profesional (SalasRuiz y Sánchez-Rivas, 2019). Aunque los autores han realizado el estudio en el sector de la salud, las ventajas de dicho uso, podrían ser aplicables a cualquier familia profesional y a cualquier contexto educativo, no solo al contexto de la FP.

\subsection{Unidad de análisis: pedagogía, recursos y evaluación}

Ya sea a nivel de la educación en general, como de forma concreta en el área de FP, el cambio más significativo en la actividad docente que puede generar la tecnología empieza por un cambio en la representación pedagógica (Anderson, 2016; Beetham y Sharpe, 2013; Chatti, Jarke y Frosch-Wilke, 2007; Loveless, 2011). El cambio educativo con tecnología no se puede entender solo como un cambio de herramienta en educación, sino como una proyección de una forma educativa de pensar el valor y la función de la tecnología en el aprendizaje en el aula (Ertmer et al, 2012; Suárez-Guerrero, 2014; Van Acker, Van Buuren, Kreijns y Vermeulen, 2011). Por esta razón, el diseño y la evaluación de los aspectos propios de la pedagogía respecto a la tecnología son clave para entender la competencia digital del docente en FP.

La pedagogía y la tecnología siguen fines y procesos distintos que, no obstante, pueden complementarse. Pero en este cruce cabe destacar dos aspectos: por un lado, la pedagogía sirve, o debería servir, como marco comprensivo para un uso adecuado y significativo de la tecnología en la educación y, por otro, el proceso de construcción de ese marco no es un proceso automático, requiere un gran esfuerzo de análisis e investigación del impacto de la tecnología tanto en nuestras vidas como en la representación de la educación. Gracias a estos esfuerzos por entender educativamente la tecnología se puede hablar de una línea de investigación y desarrollo educativo amplio (Attwell y Hughes, 2010; Koehler y Mishra, 2005; Veletsianos, 2011) donde se puede hablar de pedagogía digital (Lewin y Lundie, 2016; Morris, 2013; Turner, 2017). Este trabajo busca aprovechar este enfoque pedagógico para entender, evaluar y proponer mejoras en la competencia digital del profesorado de FP.

Tomando como base los diversos trabajos que entienden la necesidad del desarrollo pedagógico en la construcción de la Competencia Digital Docente (Esteve, 2015; Hall, Atkins y Fraser, 2014; Hatlevik, 2017; Gudmundsdottir y Hatlevic, 2018; Redecker, 2017) se puede caracterizar una definición: La Competencia Digital Docente (CDD) es una dimensión de la función docente que integra conocimientos, habilidades y actitudes que permiten a los docentes tomar decisiones sobre como enriquecer las oportunidades de aprendizaje permanente de su alumnado, así como su propio desarrollo profesional (Suárez-Guerrero, Lizandra y Ros, 2020).

Por ello, la competencia digital, vista así, es un concepto educativo y no solo tecnológico. No obstante, al hablar de CDD, cabe hacer dos apuntes: la CDD no es sinónimo de competencia digital, la primera tiene que ver con aspectos propios de la función docente y la segunda con la ciudadanía. Y, la CDD no es sinónimo de habilidad técnica, supone

Aproximación a la competencia digital docente en la formación profesional. Suárez-Guerrero, Ros-Garrido y Lizandra. 
diversas y complejas dimensiones de la función docente donde la pedagogía, los recursos y la evaluación forman un núcleo básico de representación en el trabajo en un aula.

Así, si bien es cierto que en este trabajo se exploran las seis áreas establecidas en Redecker (2017), el núcleo de análisis sobre el que se profundiza y hace la propuesta de cara a la mejora de la CDD en el contexto de la FP, se funda en un núcleo más concreto, articulado y significativo para la actividad docente que es la relación entre enfoque pedagógico, el recurso digital y la evaluación, es decir, en el cómo y con qué enseñar, y cómo valorar el aprendizaje.

\subsubsection{Pedagogía}

La pedagogía es aquí el principio rector del uso de los recursos y la evaluación (SuárezGuerrero, Lloret-Catalá y Mengual, 2016). Esta necesidad de clarificar el enfoque pedagógico es una necesidad fundamental en la comprensión y el desarrollo de la educación digital, ya que hoy en día no es posible hacer una oferta educativa plausible sin una perspectiva pedagógica sobre cómo utilizar la tecnología (Gros, 2016). La innovación educativa no sólo se basa en la novedad de la tecnología, sino más bien en el enfoque de aprendizaje que puede fomentarse con esa herramienta (Daniela, 2018). Esta necesidad de pedagogía ya ha sido subrayada por modelos como TPACK, Technology, Pedagogy and Content Knowledge (Mishra y Koehler, 2006) que permiten articular el conocimiento disciplinario, tecnológico y pedagógico al mismo nivel.

La innovación educativa con tecnología no llega solo por la novedad técnica, sino que se asienta en ideas pedagógicas más estables y validadas. Según la revisión hecha por Montanero (2019), las actuales innovaciones educativas "retoman ideas pedagógicas del siglo pasado, enriquecidas con nuevos recursos didácticos y tecnológicos" (p. 5). Así, podemos identificar cuatro orientaciones o escenarios educativos de actividad docente con tecnología: enseñanza con TIC, aprendizaje individual, aprendizaje colaborativo y autoaprendizaje del alumnado (ver Figura 1).

\subsubsection{Recursos}

Las decisiones que toma un docente sobre qué tecnología usar en su actividad docente pasan por un modelo pedagógico consciente o no. El abanico de opciones tecnológicas es muy amplio (Adams et al, 2017), que van desde la pizarra digital, las redes sociales, los recursos educativos abiertos, las bases de datos, los buscadores, el móvil, la realidad aumentada, los entornos colaborativos, los entornos virtuales de aprendizaje, los blogs, los videos, las wikis, la impresora 3D, entre otros.

Tales desarrollos nos permiten hablar sobre nuevas oportunidades no solo en el ámbito de la enseñanza sino también como una comprensión de la educación y la educación abierta (Valverde, 2010). Incluso en la FP, es relevante considerar las oportunidades en el aula generadas por los recursos educativos abiertos para una educación no formal e informal (Peters, Liu y Ondercin, 2012; Wiley, 2013).

Aunque la variedad de recursos sea exorbitante, lo importante para el análisis de la CDD es que los recursos digitales están condicionados por el enfoque pedagógico. Por tanto, dichos recursos son una segunda capa de análisis que, como se ha señalado, depende del

Aproximación a la competencia digital docente en la formación profesional. Suárez-Guerrero, Ros-Garrido y Lizandra. 
enfoque pedagógico. De nuevo, se puede identificar cuatro escenarios educativos de actividad docente (ver Figura 1).

\subsubsection{Evaluación}

La evaluación del proceso de enseñanza-aprendizaje es un elemento fundamental en todo enfoque pedagógico y que puede adquirir diferentes sentidos dependiendo del enfoque. Es necesario comprender el valor de la evaluación en el proceso y la complejidad que la tecnología incorpora a la actividad educativa. No obstante, como en el caso de la pedagogía, se puede usar la tecnología para hacer en la evaluación lo mismo de siempre, pero con tecnología (Stödberg, 2012), o para ensayar otras alternativas. El caso es que la evaluación del aprendizaje con tecnología, ya forma parte de una línea de desarrollo educativo que comparte, en esencia, los dilemas más generales de la evaluación del aprendizaje (Crisp, 2011).

Además de esto, pueden surgir nuevos objetos de valoración educativa como, por ejemplo, evaluar las habilidades comunicativas online, evaluar la capacidad de gestión la incertidumbre que genera la sobreexposición a la información o evaluar el desempeño online en equipos colaborativos (Williams, 2017). Pero, aunque se trate de nuevas herramientas, procesos sofisticados para evaluar con tecnología u objetos de análisis nuevos, hay evidencia que también en el mundo de la evaluación electrónica las actitudes docentes sobre la evaluación siguen siendo muy importantes (Adachi, Tai, y Dawson, 2018). De aquí la relevancia de conocer y analizar la evaluación junto con el modelo de representación pedagógica en el contexto de la FP. Por lo tanto, también podemos identificar cuatro escenarios evaluativos de actividad docente (ver Figura 1).

Por tanto, la propuesta que se ofrece genera cuatro orientaciones sobre la pedagogía digital, los recursos digitales y la evaluación del aprendizaje con tecnología, basadas en el aprendizaje individual (por ejemplo, videos de enseñanza), la enseñanza con TIC (por ejemplo, clase por videoconferencia), el aprendizaje colaborativo (por ejemplo, participación en foro o blog) y el autoaprendizaje del alumnado (por ejemplo, lectura de páginas web, blogs, artículos). En la figura 1 se representa la relación entre las tres dimensiones y las cuatro opciones.

Aproximación a la competencia digital docente en la formación profesional. Suárez-Guerrero, Ros-Garrido y Lizandra. 


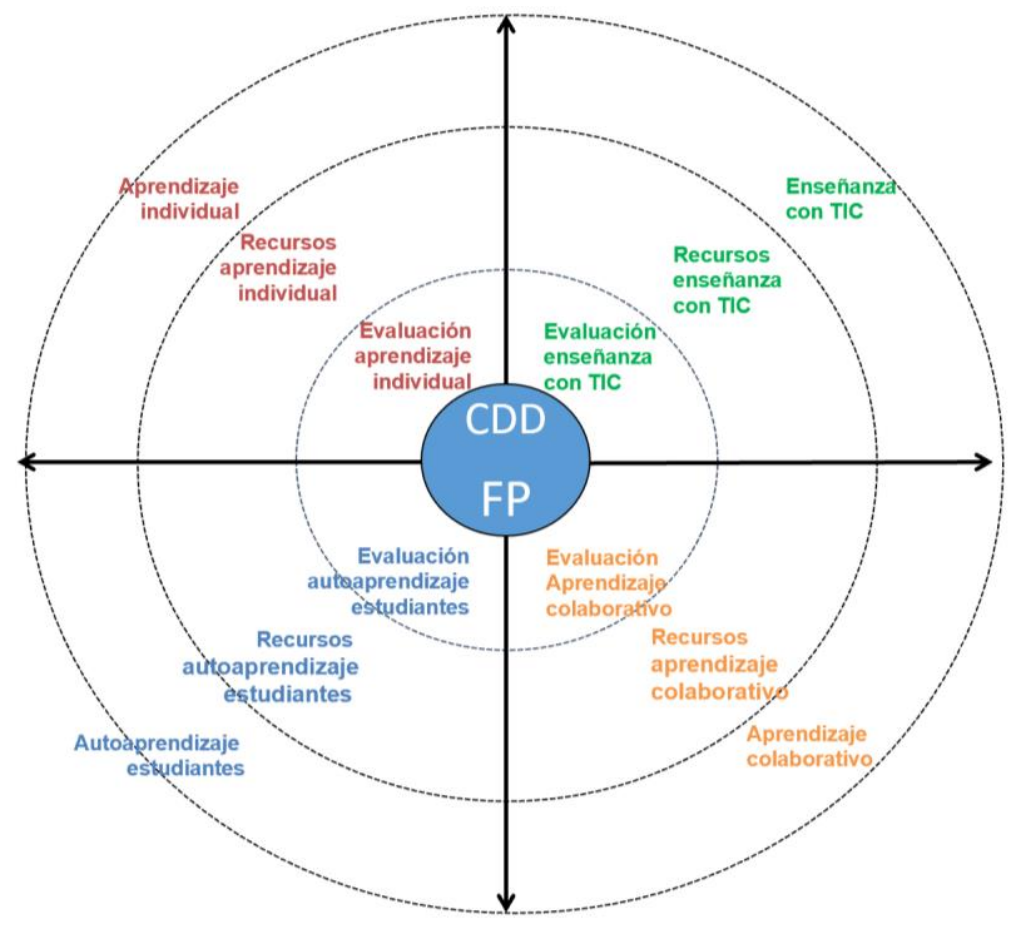

Figura 1. Orientaciones sobre la pedagogía digital, los recursos digitales y la evaluación del aprendizaje. Elaboración propia.

\section{Objetivos del estudio}

Concretamente, el objetivo es caracterizar la relación pedagogía-recursos-evaluación como elemento clave en la comprensión de la CDD del profesorado de FP a partir de la información obtenida de los centros de la provincia de Valencia (España) participantes en el estudio.

Los objetivos específicos a desarrollar son:

- Conocer la percepción del profesorado de FP acerca de las competencias digitales que tienen y de las que carecen.

- Analizar las competencias digitales que consideran necesarias en su actividad profesional.

\section{Método}

La metodología empleada se ha basado en el análisis cualitativo, dentro del paradigma interpretativo, de entrevistas realizadas a profesorado de FP de acuerdo a las orientaciones sobre la pedagogía digital, los recursos digitales y la evaluación del aprendizaje definidas en la unidad de análisis.

El equipo investigador desarrolló un guion de entrevista basándonos en los principios del marco común de referencia de competencia digital DigCompEdu propuesto por la comisión europea (Redecker, 2017). Fue discutida y acordada con los socios del proyecto TaccleVET y, una vez acordada la versión final, se procedió con la administración del

Aproximación a la competencia digital docente en la formación profesional. Suárez-Guerrero, Ros-Garrido y Lizandra. 
instrumento, en forma de entrevista semiestructurada. La entrevista constó de 23 preguntas principales, organizadas en siete secciones. La primera correspondió a datos sociodemográficos tales como edad, género, años de experiencia profesional y docente, años de experiencia como tutor de prácticas, familia profesional, organización en la que trabaja y rol asignado en dicho centro. Las seis secciones restantes correspondieron a cada una de las seis dimensiones principales del marco de competencia digital DigCompEdu: compromiso profesional, recursos digitales, pedagogía digital, evaluación, empoderamiento de alumnado y facilitación de la competencia digital del alumnado. Las entrevistas se realizaron entre febrero y mayo de 2019 teniendo una duración que oscilaba entre una hora y hora y media. La mayoría de ellas fueron grabadas con el permiso de las personas entrevistadas. Algunas de ellas ocurrieron en las escuelas de formación profesional, mientras que otras tuvieron lugar en otros lugares y fuera del horario de trabajo. En total se realizaron entrevistas a once docentes provenientes de siete centros de formación profesional de la provincia de Valencia (España). En la tabla 1 aparecen los detalles del profesorado entrevistado. La selección se realizó por conveniencia, teniendo en cuenta que se trataba de población con una variedad sustancial en cuanto a género y edad suficiente, como para obtener resultados diversos.

Tabla 1.

Características del profesorado de FP entrevistado

\begin{tabular}{ccccc}
\hline & Sexo & Edad & Familia profesional & Experiencia docente \\
\hline 1 & Hombre & $>50$ & Servicios socioculturales y a la comunidad & $>30$ \\
\hline 2 & Mujer & $>40$ & Servicios socioculturales y a la comunidad & $>7$ \\
\hline 3 & Hombre & $>50$ & Formación y orientación laboral (FOL)* & $>19$ \\
\hline 4 & Hombre & $<30$ & Servicios socioculturales y a la comunidad & $>3$ \\
\hline 5 & Hombre & $>40$ & Servicios socioculturales y a la comunidad & $>12$ \\
\hline 6 & Hombre & $>50$ & Actividades físicas y deportivas & $>28$ \\
\hline 7 & Hombre & $>30$ & Actividades físicas y deportivas & $>8$ \\
\hline 8 & Hombre & $>50$ & Servicios socioculturales y a la comunidad & $>25$ \\
\hline 9 & Mujer & $>30$ & Servicios socioculturales y a la comunidad & $>7$ \\
\hline 10 & Hombre & $>40$ & Actividades físicas y deportivas & $>18$ \\
\hline 11 & Hombre & $>40$ & Formación y orientación laboral (FOL)* & $>10$ \\
\hline
\end{tabular}

* El módulo de Formación y Orientación Laboral no se corresponde con una familia profesional, sino que se trata de un módulo común en el plan de estudios de todos los ciclos formativos de formación profesional. Fuente: Elaboración propia.

\subsection{Análisis de las entrevistas}

El análisis consistió en una interpretación cualitativa de la información obtenida en la entrevista, basada en el paradigma interpretativo. La primera fase fue reducir los datos de la entrevista a una cantidad manejable de información. Para ello, se optó por la lectura comprensiva para identificar las propiedades de los datos, conceptos e ideas de acuerdo a los aspectos pedagógicos del aprendizaje con tecnología, los recursos tecnológicos utilizados y la incorporación de la tecnología en los procesos de evaluación.

En una segunda fase, se realizó un proceso de inducción analítica mediante el cual se relacionó la información clasificada en las tres categorías principales con las cuatro orientaciones: aprendizaje individual del alumnado, enseñanza con TIC, fomento del aprendizaje colaborativo y autoaprendizaje del alumnado. El análisis permitió conocer en qué medida la visión pedagógica de la tecnología del docente, el conocimiento y

Aproximación a la competencia digital docente en la formación profesional. Suárez-Guerrero, Ros-Garrido y Lizandra. 
facilitación de recursos tecnológicos y la evaluación con tecnología, tenían un peso específico en las cuatro orientaciones señaladas.

Por tanto, este proceso analítico basado en el paradigma interpretativo es el que permitió estructurar los principales resultados, que se acompañan con algunos extractos de las entrevistas, para dar consistencia y validez a los resultados obtenidos (Martínez, 2006; Strauss y Corbin, 2002).

\section{Resultados y discusión}

En la figura 2 se muestran los resultados obtenidos de acuerdo a las cuatro orientaciones sobre la pedagogía digital, los recursos digitales y la evaluación del aprendizaje: aprendizaje individual, la enseñanza con TIC, aprendizaje colaborativo y autoaprendizaje del alumnado.

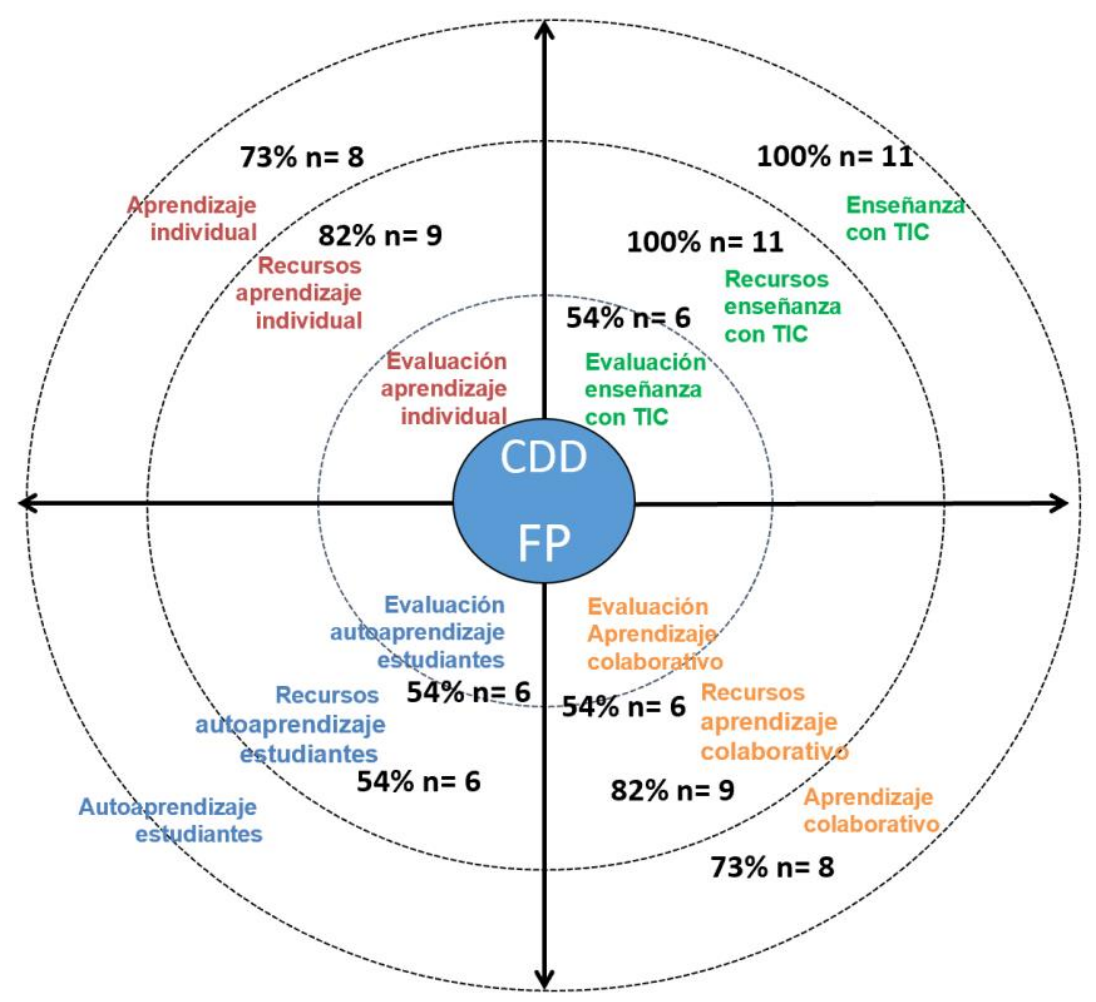

Figura 2. Resultados en las orientaciones sobre la pedagogía digital, los recursos digitales y la evaluación del aprendizaje.

En el contexto español hay 26 familias profesionales y el profesorado entrevistado pertenece a la familia de "Actividades físicas y deportivas" y "Servicios socioculturales y a la comunidad" o imparte una asignatura transversal a todos los ciclos formativos llamada "Formación y Orientación Laboral", por lo que no pertenecen a sectores en los que, a priori, la competencia digital necesaria en el sector sea muy destacada.

Aproximación a la competencia digital docente en la formación profesional. Suárez-Guerrero, Ros-Garrido y Lizandra. 
A continuación, se analiza en primer lugar los resultados obtenidos en cada uno de los cuadrantes y, a continuación, se presentan las fortalezas y buenas prácticas observadas con respecto a la competencia digital del profesorado de FP participante en la investigación. Después, se pone el foco en algunas debilidades detectadas en las categorías de análisis explicadas, pues lo que se pretende en última instancia es ofrecer algunas recomendaciones para mejorar la competencia digital del profesorado y el enfoque pedagógico de los procesos de enseñanza-aprendizaje con tecnología.

\subsection{Análisis de los cuadrantes}

El cuadrante más destacado es el relacionado con la enseñanza con TIC, donde todo el profesorado coincide en el uso de tecnología para la enseñanza y de recursos digitales. Pero no todos coinciden en la evaluación del aprendizaje con tecnologías. Así, el profesorado es muy consistente en su propuesta, ya que articula de manera compensada, tanto el enfoque pedagógico, con el uso de recursos y la evaluación. Se puede decir que es el cuadrante en el que se muestra más competente, dada tanto la frecuencia como la consistencia.

En el segundo cuadrante a destacar es el referido al aprendizaje colaborativo, donde el profesorado se muestra consistente en su propuesta pedagógica y el uso de los recursos. De nuevo, no hay consistencia en el ámbito de la evaluación, pues la frecuencia disminuye. Por tanto, el segundo ámbito en el que se muestra más competente el profesorado de FP es en la articulación de la colaboración en su enfoque pedagógico con el uso de los recursos, pero no tanto en su propuesta de evaluación. En esta ocasión, según la frecuencia y la consistencia observada, se puede decir que el profesorado sería medianamente competente en el cuadrante del aprendizaje colaborativo.

El tercer cuadrante a destacar es el referido al aprendizaje individual con TIC, donde la mayoría del profesorado articula bien el enfoque pedagógico con el uso de recursos. Sin embargo, ningún docente considera el uso de tecnologías en la evaluación del aprendizaje individual, aspecto llamativo puesto que sí la consideraba para el aprendizaje colaborativo. Por tanto, es un cuadrante parcialmente consistente, ya que exhibe una carencia total en el uso de las TIC para la evaluación.

El cuadrante con menor presencia es el referido al autoaprendizaje del alumnado, donde poco más de la mitad del profesorado considera la incorporación de lo digital en su enfoque pedagógico y en el uso de recursos para el mismo el autoaprendizaje. De nuevo, ningún docente considera el uso de tecnologías en la evaluación de esta orientación. Es el cuadrante más deficitario donde destaca la frecuencia más baja y solo hay consistencia entre la propuesta pedagógica y el uso de recursos, pero no en la propuesta de evaluación, que es inexistente.

Teniendo en cuenta la consistencia y la frecuencia, la competencia del docente es: altamente consistente en el ámbito de la enseñanza con TIC y exhibe una alta práctica; medianamente consistente en el uso de la tecnología en el ámbito del aprendizaje colaborativo pero una alta práctica; poca consistencia en el uso de la tecnología en el ámbito del aprendizaje individual pero alta práctica; y es nula la consistencia en el uso de tecnologías en el ámbito del autoaprendizaje y muy poca práctica. Por tanto, el nivel de competencia del docente de FP es alto en el ámbito de la enseñanza con TIC; medio en el

Aproximación a la competencia digital docente en la formación profesional. Suárez-Guerrero, Ros-Garrido y Lizandra. 
uso de la tecnología en el ámbito del aprendizaje colaborativo; poco en el uso de la tecnología en el ámbito del aprendizaje individual; y se podría considerar carente de competencia en el uso de tecnologías en el ámbito del autoaprendizaje. En la figura 3 se muestra la proporción de los niveles de competencia en cada una de las orientaciones.

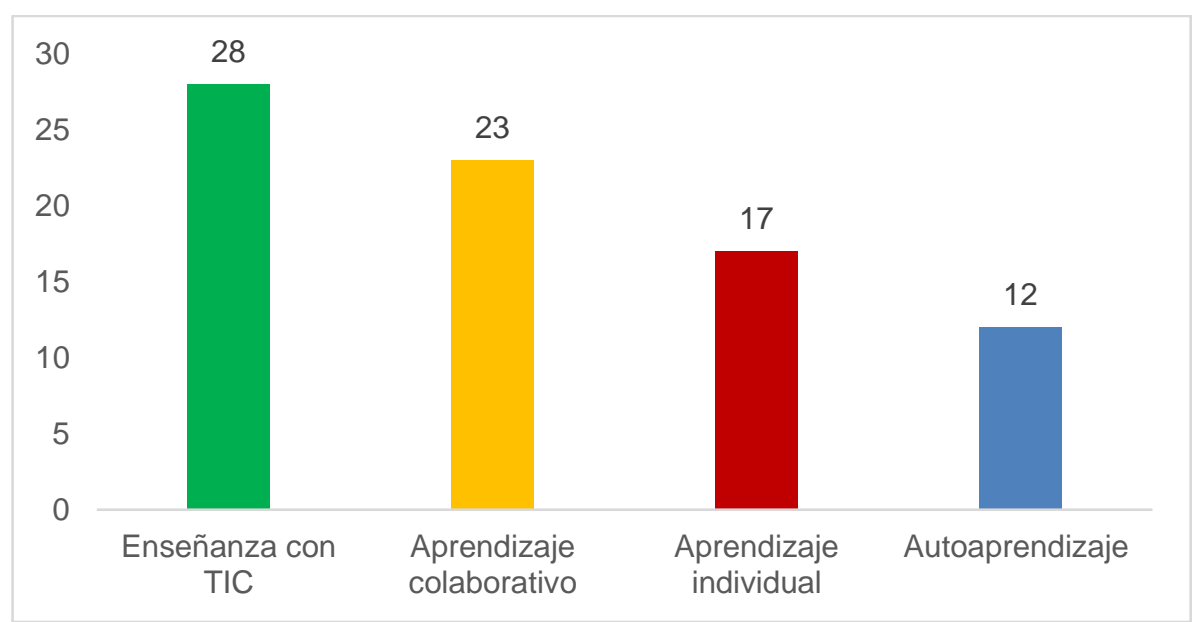

Figura 3. Representación del nivel de competencia en cada una de las orientaciones a partir de las interacciones obtenidas de la información proporcionada por el profesorado.

\subsection{Análisis de las fortalezas y buenas prácticas}

Después del análisis de los resultados obtenidos en cada uno de los cuadrantes vamos a reflexionar sobre tres fortalezas y buenas prácticas que se han observado en el profesorado entrevistado: sobre el conocimiento y nivel de competencia digital del profesorado; sobre los recursos para el aprendizaje individual y para el aprendizaje colaborativo en general y durante las clases; y, por último, reflexionaremos sobre el enfoque analógico de la enseñanza con tecnología. Para ello se extraerán fragmentos de las entrevistas realizadas que ejemplifican el análisis.

Se ha observado un conocimiento y nivel de competencia digital heterogéneo en el profesorado entrevistado, siendo una minoría el que declaraba y mostraba un alto conocimiento en competencia digital. No obstante, se observó una buena predisposición y una actitud positiva del profesorado a mejorar su competencia digital. De hecho, en todos los casos se observó que la tecnología estaba presente en mayor o menor medida en sus prácticas docentes, así como una intención explícita a seguir incorporándola.

Además, es evidente que el profesorado entrevistado conoce y hace uso de recursos tecnológicos en su desarrollo profesional. Concretamente, el profesorado utiliza la tecnología para resolver problemas en los procesos de enseñanza, para los que consideran que puede ser útil. Un docente nos dijo:

"me doy cuenta de que, si les pido una tarea escrita, la copian. No obstante, mediante la edición de videos, ellos realizan todo el proceso. Utilizo la tecnología por este motivo" (Docente 10). 
Así, un claro ejemplo es la tendencia a sustituir algunos de los trabajos escritos que habitualmente pedían a su alumnado por la edición de videos explicativos sobre los contenidos específicos de algunas materias. Con esta práctica pretenden, por un lado, evitar que los trabajos teóricos continúen siendo un compendio de información copiada de internet, así como estimular sus capacidades creativas y su motivación hacia el aprendizaje. Además, se mejora el desarrollo de sus competencias de aprendizaje de la materia, así como las competencias de aprender a aprender o la propia competencia tecnológica. Es decir, tratan de instruir al alumnado en la búsqueda y selección de información relevante y que está disponible en la red, estimulando la participación activa del alumnado, pues es una actitud valorada en el futuro ámbito profesional.

También se ha observado que determinadas plataformas atraen más al alumnado (Facebook o Yammer). Esta manera de mostrar los contenidos no solo favorece el aprendizaje individual o colaborativo, sino que estimulan el autoaprendizaje a la vez que logran atender algunas diferencias y dificultades en los procesos de aprendizaje. Otro docente nos decía que:

"a los estudiantes les gusta mi perfil de Facebook por los materiales y recursos que alli encuentran. De hecho, en algunos casos lo utilizo como una extensión de la clase presencial. Además, hay estudiantes con menor nivel que aprovechan la información que vuelco en la red o en aplicaciones como Yammer. Del mismo modo, hay estudiantes que carecen de la seguridad y autoconfianza para pedir ayuda directamente en la clase presencial. En cambio, mediante el correo electrónico o herramienta de Yammer pueden hacerlo con mayor seguridad" (Docente 4).

Con respecto al uso de recursos en clase, el profesorado de FP entrevistado coincide en afirmar que existe un amplio, incluso a veces excesivo catálogo de dispositivos y recursos tecnológicos. El profesorado también declara que el uso de internet o las redes sociales puede ser muy útil, así como la presencia del teléfono móvil en clase. De hecho, en los casos en los que el alumnado muestra un bajo nivel competencias para la búsqueda de información, el docente les ayuda en clase para que posteriormente pueda hacerlo con mayor competencia y autonomía.

No obstante, se detectó en las entrevistas cómo se le atribuye un enfoque analógico a la enseñanza con tecnología. Es decir, algunos docentes desarrollan prácticas que habitualmente realizaban sin el apoyo de la tecnología, con dispositivos tecnológicos, lo que coincidiría con la aportación de Stödberg (2012), sin que ello suponga un valor añadido destacable. De hecho, un docente nos dijo de manera explícita:

"yo habitualmente hago lo mismo que hacía sin tecnología. Solamente que con el uso de las TICs pueden hacer las tareas online" (Docente 9).

Aunque la mayoría del profesorado tiene dificultades para seleccionar los recursos tecnológicos que se adecuan a la práctica que quieren llevar a cabo, también encontramos a docentes con un gran dominio de los recursos tecnológicos:

Aproximación a la competencia digital docente en la formación profesional. Suárez-Guerrero, Ros-Garrido y Lizandra. 
"todos los contenidos están estructurados en OneNote y en las aplicaciones del entorno Microsoft. Nosotros también utilizamos "Miniprofes360" para que los estudiantes aprendan anatomía. Ellos crean los contenidos, respectando la autoría original. También se les proporciona la posibilidad de acceder a un proceso de aprendizaje personalizado mediante recursos metodológicos como la clase invertida o flipped classroom. De esta manera, tiene la posibilidad de acceder al contenido donde y cuando quieran. De hecho, les proporcionamos algunas clases a través de videoconferencia, en las que los estudiantes pueden contactar tanto en directo, como a posterior con el profesorado o sus compañeros. El trabajo con foros o chats, no solo amplía las posibilidades de tener feedback del profesorado, sino que facilita el trabajo colaborativo. No obstante, el trabajo colaborativo se lleva a cabo de manera presencial en el aula también" (Docente 7).

Insistimos que esta no es la tónica habitual, pero de alguna manera debería ser un estándar al que habría que aspirar si verdaderamente se quiere hacer uso de la tecnología aprovechando las posibilidades pedagógicas que tiene.

\subsection{Análisis de debilidades detectadas}

Habiendo analizado las fortalezas, el análisis de las entrevistas permitió detectar al menos tres debilidades en el uso pedagógico de la tecnología en la formación profesional.

La primera es que, generalmente, es limitada la variedad de recursos y aplicaciones tecnológicas conocidas y utilizadas por el profesorado. En ocasiones, están limitados también por el contexto educativo que tiene recursos limitados (como el propio acceso a internet a determinadas páginas web que es complicado en algunos centros). Aunque también hay profesorado que apenas llega a usar los recursos disponibles (como la propia plataforma virtual) por desconocimiento de cómo funciona. Además, en algunos casos las prácticas difieren poco del uso convencional que se realiza en el plano personal, es decir, para la búsqueda y gestión de información y para comunicarse a distancia. Esto es debido a que la mayoría del profesorado entrevistado se reconoce como perfil de baja competencia digital, hecho que les genera inseguridad en la incorporación de recursos y posibilidades tecnológicas que no saben manejar con certeza. De hecho, una profesora nos declara lo siguiente:

"yo soy un poco tecnófoba, por lo que necesito mucho apoyo para utilizar la tecnología, por lo que carezco de la confianza suficiente para incorporar nuevos recursos. Aunque soy consciente del gran potencial que tienen, en mi caso necesito que las prácticas sean simples, pues si algo funciona mal con la tecnología, corro el riesgo de que la lección sea un fracaso, debido a mi insuficiente competencia digital" (Docente 2).

El uso de dispositivos o recursos digitales no implica un uso y desarrollo de la competencia digital docente, ni que se esté formando o desarrollando la competencia digital del estudiantado. Es importante destacar que la manera de utilización de los recursos muestra la propia concepción pedagógica del profesorado ya que, en algunos docentes, sigue primando un uso tradicional y técnico instrumental que se aleja de la concepción práctica, aunque en ocasiones sí se vislumbra una concepción crítica de dichos recursos.

Aproximación a la competencia digital docente en la formación profesional. Suárez-Guerrero, Ros-Garrido y Lizandra. 
El segundo problema se fundamente en la concepción socialmente aceptada del concepto nativo digital. Este término, popularizado entre otros por Marc Prensky (2001), apoya la idea de que la gente joven, por el hecho de serlo y haber nacido y crecido en la era digital, cuenta con una cultura digital amplia. De hecho, parte del profesorado admitía que sentía que, algunos casos, la competencia digital del estudiantado era superior a la suya. No obstante, el hecho que los estudiantes hayan nacido en la era digital, solamente supone que ellos tienen una mayor facilitad para el acceso o el dominio técnico de la tecnología, pero carece de rigor empírico (Bullen, Morgan y Qayyum, 2011; Kirschner y De Bruyckere, 2017). En cambio, aun asumiendo que esto fuera cierto, que no siempre ocurre, los y las estudiantes carecen en la mayoría de los casos de un conocimiento amplio sobre las posibilidades que determinados recursos tecnológicos tienen, no solo en el plano social sino por ejemplo en el académico. De hecho, una profesora nos decía en la entrevista que:

"hay estudiantes con alta competencia tecnológica, pero es difícil para ellos aplicar esa competencia al ámbito académico o profesional. Por ejemplo, es complicado para ellos colaborar en la red. Por tanto, como docentes en formación profesional, si nos formamos de manera adecuada, tenemos mucho que aportar en ese sentido, pues probablemente sepan más que los docentes sobre aplicaciones y utilidades técnicas, pero nosotros podemos y debemos estar preparados para resolver sus dudas, motivarlos y guiarlos hacia usos relevantes de la tecnología" (Docente 6).

Por tanto, parece que partir de la premisa de que los jóvenes estudiantes o incluso el profesorado más joven tienen de partida una mejor competencia digital por el hecho de ser jóvenes, no solo es problemático, sino que es una idea reduccionista que no se demuestra en la práctica habitual.

La tercera debilidad gira en torno a la falta de estrategias para incorporar la tecnología en los procesos de evaluación declarada por el profesorado entrevistado. Se observó de manera generalizada que el profesorado no solo no muestra competencia en ese sentido, sino que tampoco mostró mucho compromiso para utilizar la tecnología como recurso de evaluación. De hecho, en los casos en los que se afirmaba utilizarla, se ha visto cómo la tecnología se utilizaba como un medio para perpetuar la evaluación como un elemento tradicional de control o validación. No se vio en ningún caso que se utilizara la posibilidad de interacción en red para proponer procesos de evaluación basados en la colaboración, la evaluación por pares o la autoevaluación de conocimientos. De hecho, el estudio de Marhuenda-Fluixá, Chisvert-Tarazona, Palomares-Montero y Vila (2017), realizado en el contexto de la FP Dual pero extensible a la FP en general, mostró cómo los usos más extendidos de la tecnología como medio de evaluación eran la corrección de trabajos, las técnicas de observación, el desarrollo de pruebas objetivas o pruebas de ejecución. Tan solo las pruebas objetivas podrían implicar el uso de algún recurso digital. En cambio, los instrumentos menos utilizados fueron el uso del portafolio como repositorio de trabajos realizados y la autoevaluación. Estos instrumentos podríamos suponer que implicarían un uso de tecnologías. Pero consideramos que la utilización de todos los instrumentos listados es más instrumental que un reflejo de su propia competencia digital o de la pretensión de desarrollar la competencia digital del estudiantado.

Aproximación a la competencia digital docente en la formación profesional. Suárez-Guerrero, Ros-Garrido y Lizandra. 
Las tecnologías en el proceso de enseñanza-aprendizaje llevan implícito la automatización y la simplificación de la información, ayudan a compartir, distribuir, recoger información, entre otros aspectos. Ayudan durante el proceso. Pero el profesorado, en general, es reacio a utilizarla en la evaluación final. Quizá sea positivo la ausencia de las tecnologías en la evaluación si estas se convierten en una comprobación final (por ejemplo, un test de opciones que la propia plataforma te da los resultados numéricos obtenidos). Habrá que pensar en posibilidades para evaluar directamente relacionadas con el desarrollo de competencia digital o ¿la introducción de las tecnologías en la evaluación estaría reproduciendo el paradigma técnico-tradicional (ya superado en el desarrollo de la docencia)? Quizá el profesorado es más reacio al uso de las tecnologías en la evaluación final porque en la evaluación del proceso las utiliza en mayor medida con un fin instrumental de controlar el qué se enseña y aprende utilizando un cómo (recursos digitales). Quizá, sea más pedagógico la ausencia de la tecnología en la evaluación final asimilándola a la calificación, entendida como recurso final pero que no está reñida con el uso de recursos digitales en el proceso de enseñanza-aprendizaje que permiten acercarse en mayor medida a una "verdadera" evaluación. Quizá la confusión es entre calificación y evaluación (Hernández y Salinas 2008).

Por último, para que la evaluación con tecnología tenga sentido pedagógico se requiere de un enfoque que difiera de la evaluación tradicional. Además de valorar qué recursos o dispositivos tecnológicos permiten evaluar, la idea angular siempre es el enfoque y sentido de la evaluación. No obstante, a este ya problemático trabajo, cabría considerar que la evaluación con tecnología puede ir más allá de la mera validación de adquisición de contenidos y que podría facilitar nuevos dominios de acción u objetos de evaluación, como las habilidades comunicativas en línea, la gestión de la incertidumbre en el aprendizaje debido a la cantidad de información disponible en la red, o para aprovechar las posibilidades que la red permite para que el alumnado coopere en las actividades de aprendizaje (Williams, 2017).

\section{Conclusiones}

En el Proyecto TaccleVET se trabajó sobre la forma en que el profesorado se percibe como competente digital. Las entrevistas han servido para observar la pedagogía, los recursos y la evaluación en su función integral, pero donde la pedagogía sirve de hilo conductor en torno a la cual se integran todas las demás. Como se puede ver en os resultados, hay profesorado que posee un nivel alto de competencia, pero es consciente de que debe mejorar y, por otra parte, hay profesorado que cree que tiene un nivel competencial mayor del que tendría porque parte de una visión más instrumental y utilitarista de la tecnología. No obstante, este análisis debe realizarse teniendo como marco que el profesorado entrevistado pertenece a sectores profesionales donde la competencia digital no es un aspecto imprescindible. Como en otros muchos análisis sobre la profesión docente, se puede señalar que la competencia digital del profesorado depende en mayor medida de la propia iniciativa, formación y experiencia del propio profesorado que de las prácticas educativas sistemáticas en un determinado sector profesional. Sorprende que el profesorado más experimentado en la docencia y, por lo tanto, de mayor edad, está más abierto y es menos reacio a incorporar las competencias digitales en los procesos de enseñanza-aprendizaje. La comprensión pedagógica global

Aproximación a la competencia digital docente en la formación profesional. Suárez-Guerrero, Ros-Garrido y Lizandra. 
de su oficio es el aspecto que da sentido y significado a la incorporación de una perspectiva digital (Lizandra, Ros-Garrido, Suárez-Guerrero, y Marhuenda, 2019). Aunque también existe profesorado joven y con menos experiencia docente, pero con una amplia formación que le acerca a una elevada competencia digital. La comprensión pedagógica general de su oficio es el aspecto relevante que da sentido y significado a la incorporación de una perspectiva digital. La pedagogía es el hilo conductor, la que determina el sentido y el enfoque del uso de los recursos y la evaluación. Todo el profesorado entrevistado tiene claro que los recursos son sólo recursos y que todo depende del enfoque pedagógico que confiere un sentido u otro a los recursos y a la evaluación, dotándoles de mayor o menor utilidad y relevancia en el proceso de enseñanzaaprendizaje. Por tanto, la primera conclusión de la investigación es que la competencia digital docente no está relacionada con la edad del profesorado y obedece más a opciones personales de su manera de concebir su profesionalidad docente.

Con relación al primer objetivo específico del estudio (conocer la percepción del profesorado de FP acerca de las competencias digitales que tienen y de las que carecen), los resultados muestran que el profesorado conoce y administra algunas metodologías y recursos tecnológicos, pero no incorpora la tecnología a los procesos de evaluación. Quizá se deba a no sólo a cuestiones técnicas sino también éticas (Floridi, 2013) y donde los criterios de evaluación de las asignaturas impartidas no contemplan la competencia digital ni conciben que las herramientas digitales puedan mejorar la evaluación de los procesos. Sin embargo, la mayoría del profesorado entrevistado considera que hay un amplio margen para comprender mejor la evaluación como una práctica educativa más que como una práctica punitiva, una visión que el uso de herramientas digitales puede mejorar. Otra posible explicación esté en la raíz de qué es lo que se evalúa, que está relacionado con el cómo se evalúa, ambas dimensiones se interrelacionan. También cabe pensar en el sentido otorgado a la evaluación entendiéndola como calificación (evaluación final o sumativa) y no como evaluación del proceso (evaluación procesual o formativa). Así, uno de las principales reflexiones docentes debería ser valorar qué recursos o dispositivos tecnológicos permiten evaluar aquello que pretendemos evaluar, es decir, es necesario profundizar en qué es lo que se considera que debe evaluarse en los procesos de enseñanza-aprendizaje en la FP y recuperar el sentido de una evaluación formativa. A lo largo del proceso sí parece que incorporan recursos digitales tanto en los procesos de aprendizaje individual como grupal. Pero es necesario diferenciarla de la evaluación sumativa, dónde se evidencia el sentido de la calificación por encima de la evaluación. Como segunda conclusión se puede señalar que el profesorado tiene conocimiento de algunos recursos y aplicaciones tecnológicas, actitud y capacidad para incorporarlos a su práctica docente, pero no llega a concebir la integración de la tecnología en la evaluación (entendida como evaluación final, sumativa o calificación final).

Con relación al segundo objetivo específico (analizar las competencias digitales que consideran necesarias en su actividad profesional), los resultados muestran que el profesorado conoce y gestiona los recursos de las TIC para la enseñanza y utiliza recursos concretos tanto en el aprendizaje individual como para el colaborativo e, incluso, también está presente en la evaluación del aprendizaje colaborativo. Sin embargo, no brindan oportunidades de evaluación para el aprendizaje individual ni para la autoevaluación del alumnado. Por lo que la tercera conclusión apunta a que la competencia digital se desarrolla en mayor medida en procesos de enseñanza-aprendizaje colectivos que en los

Aproximación a la competencia digital docente en la formación profesional. Suárez-Guerrero, Ros-Garrido y Lizandra. 
procesos de aprendizaje individuales. De nuevo aquí podemos recuperar el qué se evalúa y el significado que comparten de evaluación confundiéndolo, quizá, con calificación.

En definitiva, el trabajo tenía como objetivo una aproximación a la comprensión, identificación y caracterización de la relación pedagogía-recursos-evaluación como elemento clave en la comprensión de la CDD del profesorado de FP, a partir del estudio de los casos de los once informantes participantes en el presente estudio limitado a la provincia de Valencia (España). Para seguir mejorando el prestigio y calidad de la FP es necesario, entre otros aspectos, prestar atención al profesorado que la imparte. Y la competencia digital también forma parte hoy en día del sentido de ser docente. El profesorado de FP debe cuidar su doble profesionalidad técnico-profesional y de carácter pedagógico-didáctica (Ros-Garrido y Marhuenda-Fluixá, 2019). No basta saber, sino que también hay que saber enseñarlo, las tecnologías y los recursos digitales son herramientas imprescindibles hoy en día en cualquier ámbito y contexto educativo y, más especialmente en el contexto de la FP cuyos avances técnicos y tecnológicos en la industria deben formar parte de los contextos de enseñanza-aprendizaje (especialmente en algunos sectores ocupacionales). Los cambios rápidos incrementan la vulnerabilidad docente ante su propia competencia digital, lo que provoca que el profesorado prefiera utilizar herramientas y recursos conocidos, ya sean de producción propia o ajena, especialmente para fomentar la creatividad y habilidades del alumnado. Por tanto, es clave la concienciación técnica, la concienciación pedagógica y la competencia digital. También, como planteaba Ibarrola (2009), es fundamental responder a las demandas del trabajo actual requiere modificar las estrategias didácticas, los recursos, la evaluación y acreditación de la FP. Es decir, los procesos de enseñanza-aprendizaje deberían adaptarse al alumnado actual (jóvenes con fracaso escolar y al alumnado con interés y motivación en su formación, a las personas con discapacidad, a las personas desempleadas de larga duración que vuelven al sistema educativo, a personas o grupos con especiales dificultades de integración, a las personas en situación de exclusión social o riesgo de padecerla). Y esta adaptación pasa por la CDD. Esta limitación generalizada, coloca la evaluación con tecnología como una de las dimensiones clave sobre la que actuar para mejorar el marco de competencia digital docente. Lo que es innegable es que lo digital obliga a una reestructuración de la didáctica en las clases, del diseño, desarrollo y evaluación del currículo. Queda pendiente el uso de las tecnologías en la calificación.

\subsection{Limitaciones del estudio}

La investigación ha sido realizada bajo el enfoque de análisis cualitativo sobre once entrevistas realizadas a profesorado de FP de la provincia de Valencia. Por lo tanto, constituye una primera aproximación al contexto de estudio y, aunque consideramos que los resultados pueden tener cierta transferibilidad, convendría ampliar la muestra en posteriores investigaciones para poder corroborar $y$, en su caso, generalizar las conclusiones del estudio realizado. En ese sentido, también consideramos que podría ser interesante ampliar el estudio con un diseño mixto en el que se pudiera incorporar información de carácter cuantitativo y triangular los resultados para validar el propio constructo teórico que sostiene el análisis de la competencia digital docente en este colectivo. Igualmente, creemos que la CDD no está relacionada con el sector profesional directamente, por lo que sería necesario investigar sobre otras familias profesionales para comprobar si la competencia digital docente del profesorado de FP está relacionada con el sector profesional o no. También la naturaleza de la FP invita a analizar los recursos

Aproximación a la competencia digital docente en la formación profesional. Suárez-Guerrero, Ros-Garrido y Lizandra. 
(qué), la pedagogía (cómo) y el valor del aprendizaje (evaluación) de las personas que tutorizan al alumnado en sus prácticas en las empresas.

\section{Agradecimientos}

Agradecemos al profesorado que accedió a participar como informante en este estudio, así como su disposición a seguir colaborando la autora y autores del trabajo.

Presentación del artículo: 11 de junio de 2020

Fecha de aprobación: 16 de julio de 2021

Fecha de publicación: 30 de septiembre de 2021

Suárez-Guerrero, C., Ros-Garrido, A., y Lizandra, J. (2021). Aproximación a la competencia digital docente en la formación profesional. RED. Revista de educación a distancia, 21(67). http://dx.doi.org/10.6018/red.431821

\section{Financiación}

Este trabajo se ha realizado dentro del periodo de participación en el proyecto europeo titulado "Extend European Framework for the Digital Competence of Educators for VET teachers and trainers" (TACCLE-VET) (2018-1-PT01-KA202-047324), financiado por la Comisión Europea mediante el Programa Erasmus+ KA202, Strategic Partnership for Vocational Education and Training, del que la Universitat de València fue una de los socios del consorcio.

\section{Referencias}

Adachi, C., Tai, J. H. M., \& Dawson, P. (2018). Academics' perceptions of the benefits and challenges of self and peer assessment in higher education. Assessment $y$ Evaluation in Higher Education, 43(2), 294-306. doi: https://doi.org/10.1080/02602938.2017.1339775

Adams, S., Cummins, M., Davis, A., Freeman, A., Hall Giesinger, C., \& Ananthanarayanan, V. (2017). NMC Horizon Report: 2017 Higher Education Edition. Austin, Texas: The New Media Consortium.

Ala-Mutka, K., Punie, Y., \& Redecker, C. (2008). Digital competence for lifelong learning. Sevilla: Institute for Prospective Technological Studies (IPTS), JRC. Disponible en ftp://ftp.jrc.es/pub/EURdoc/EURdoc/JRC48708.TN.pdf

Ananiadou, K., \& Claro, M. (2009). 21st century skills and competences for new millennium learners in OECD countries, EDU Working paper no. 41.

Anderson, T. (2016). Theories for Learning with Emerging Technologies. In G. Veletsianos (Ed.), Emergence and Innovation in Digital Learning: Foundations and Applications (pp. 35-50). Edmonton, AB: Athabasca University Press.

Attwell, G. \& Hughes, J. (2010). Pedagogic approaches to using technology for learning: Literature review. Wales: Pontydysgu: Lifelong Learning UK. Disponible en http://dera.ioe.ac.uk/2021/

Aproximación a la competencia digital docente en la formación profesional. Suárez-Guerrero, Ros-Garrido y Lizandra. 
Badia, A. (2015). Research trends in technology-enhanced learning, Journal for the Study of Education and Development, 38(2), 253-278. doi: http://dx.doi.org/10.1080/02103702.2015.1016744

Beetham, H., \& Sharpe, R. (Eds.). (2013). Rethinking pedagogy for a digital age: Designing for 21 st century learning (2nd ed.). London: Routledge.

Bullen, M., Morgan, T., \& Qayyum, A. (2011). Digital learners in higher education: Generation is not the issue. Canadian Journal of Learning and Technology/La revue canadienne de l'apprentissage et de la technologie,37(1), 1-24.Carretero, S., Vuorikari, R. \& Punie, Y. (2017). DigComp 2.1. The digital Competence Framework for Citizens with eight proficiency levels and examples of use. Publications Office of the European Union. Disponible http://publications.jrc.ec.europa.eu/repository/bitstream/JRC106281/webdigcomp2.1pdf_(online).pdf

Cedefop (2020). Vocational education and training in Europe, 1995-2035: scenarios for European vocational education and training in the 21st century. Luxembourg: Publications Office of the European Union. Cedefop reference series; No 114. Disponible en: http://data.europa.eu/doi/10.2801/794471

Chatti, M.A., Jarke, M., \& Frosch-Wilke, D. (2007). The future of e-learning: a shift to knowledge networking and social software. International journal of knowledge and learning, 3(4-5), 404-420. DOI: https://doi.org/10.1504/IJKL.2007.016702

Cobo, C. (2019). Acepto las condiciones. Usos y abusos de las tecnologías digitales. Madrid: Fundación Santillana.

Crisp, G. (2011). Teacher's Handbook on e-Assessment. Disponible en: http://transformingassessment.com/sites/default/files/files/Handbook_for_teachers.pd $\mathrm{f}$

Daniela, L. (Ed.) (2018). Didactics of smart pedagogy: Smart pedagogy for technology enhanced learning Cham: Springer. doi: http://dx.doi.org/10.1007/978-3-030-015510

Ertmer, P. A., Ottenbreit-Leftwich, A. T., Sadik, O., Sendurur, E., \& Sendurur, P. (2012). Teacher beliefs and technology integration practices: A critical relationship. $\begin{array}{llll}\text { Computers } y & \text { education, } & \text { 59(2), } 23-435 .\end{array}$ https://doi.org/10.1016/j.compedu.2012.02.001

Esteve, F. (2015). La competencia digital docente. Análisis de la autopercepción y evaluación del desempeño de los estudiantes universitarios de educación por medio de un entorno 3D (tesis doctoral). Disponible en: http://hdl.handle.net/10803/291441

European Commission (2010). A Digital Agenda for Europe. Disponible en: https://eurlex.europa.eu/LexUriServ/LexUriServ.do?uri=COM:2010:0245:FIN:EN:PDF

Fabregat Pitarch, A., y Gallardo Fernández, I. M. (2019). Desarrollo de competencias digitales para emprendedores desde un aula de Formación Profesional. En E. Sánchez, J. Ruiz y E. Sánchez, Innovación y tecnología en contextos educativos (790-800). Málaga: UMA editorial. Disponible en: http://roderic.uv.es/handle/10550/71885

Fainholc, B., Nervi, H., Romero, R., \& Halal, C. (2015). La formación del profesorado y el uso pedagógico de las TIC. Revista De Educación a Distancia (RED), 38. Disponible en: https://revistas.um.es/red/article/view/234081

Ferrari, A. (2013). DIGCOMP: A framework for developing and understanding digital competence in Europe. Seville. doi: https://doi.org/10.2788/52966

Floridi, L. (2013). The Ethics of Information. Oxford: Oxford University Press.

Gisbert, M., y Esteve, F. (2011). Digital Learners: la competencia digital de los

Aproximación a la competencia digital docente en la formación profesional. Suárez-Guerrero, Ros-Garrido y Lizandra. 
estudiantes universitarios. La cuestión universitaria, 7, 48-59. Recuperado de http://polired.upm.es/index.php/lacuestionuniversitaria/article/view/3359/3423

Gisbert, M.; Esteve, V. y Lázaro, J.L. (eds.) (2019) ¿Cómo abordar la educación del futuro? Conceptualización, desarrollo y evaluación desde la competencia digital docente. Barcelona: Octaedro.

Goodfellow, R., \& Lea, M (2016). Literacy and the digital university, en C. Haythornthwaite, R. Andrews y J. Fransman, The SAGE Handbook of e-learning Research (423-442). London: SAGE Publications Ltd.

Gros, B. (2016). The Dialogue Between Emerging Pedagogies and Emerging Technologies. Springer Berlin Heidelberg. Disponible en: http://link.springer.com/chapter/10.1007/978-3-662-47724-3_1

Gudmundsdottir, G.B. \& Hatlevic, O.E. (2018). Newly qualified teachers' professional digital competence: implications for teacher education. European Journal of Teacher Education, 41(2), 214-231. doi: https://doi.org/10.1080/02619768.2017.1416085

Hall, R., Atkins, L., \& Fraser, J. (2014). Defining a self-evaluation digital literacy framework for secondary educators: The digilit lecister project. Research in Learning Technology, 22. doi: http://dx.doi.org/10.3402/rlt.v22.21440

Hatlevik, O.E. (2017). Examining the relationship between teachers' self-efficacy, their digital competence, strategies to evaluate information and use of ICT at school. Scandinavian Journal of Educational Research, 61(5), 555-567. doi: https://doi.org/10.1080/00313831.2016.1172501

Hernández, R. y Salinas, B. (2008): La evaluación didáctica como componente del diseño/desarrollo curricular, en A. De la Herrán y J. Paredes: Didáctica general: la práctica de la enseñanza en educación infantil, primaria y secundaria (pp. 235-248). Madrid: McGraw-Hill.

Ibarrola, M. (2009). Formación de profesionales de la ETP. En F. Blas, y J. Planells (Eds.), Retos actuales de la educación técnico-profesional (pp. 73-88). Madrid: OEI, Fundación Santillana.

ISTE. (2008). National educational technology standards for teachers. Washington DC: International Society for Technology in Education.

Kirschner, P., \& De Bruyckere, P. (2017). The myths of the digital native and the multitasker, Teaching and Teacher Education, 67, 135-142. doi: http://dx.doi.org/10.1016/j.tate.2017.06.001

Koehler, M.J., \& Mishra, P. (2005). What happens when teachers design educational technology? The development of technological pedagogical content knowledge. Journal of Educational Computing Research, 32(2), 131-152. doi: https://doi.org/10.2190/0EW7-01WB-BKHL-QDYV

Lewin, D., \& Lundie, D. (2016). Philosophies of digital pedagogy. Studies in Philosophy and Education, 35(3), 235-240. doi: http://dx.doi.org/10.1007/s11217-016-9514-7

Lizandra, J., Ros-Garrido, A., Suárez-Guerrero, C., \& Marhuenda, F. (2019). Digital competence of VET teachers: Illustrations from non-technological professions. In B. E. Stalder \& C. Nägele (Eds.) Trends in vocational education and training research, Vol. II. Proceedings of the European Conference on Educational Research (ECER), (pp.250-258). Vocational Education and Training Network (VETNET). doi: https://doi.org/10.5281/zenodo.3371515

Loveless, A. (2011). Technology, pedagogy and education: reflections on the accomplishment of what teachers know, do and believe in a digital age. Technology,

Aproximación a la competencia digital docente en la formación profesional. Suárez-Guerrero, Ros-Garrido y Lizandra. 
Pedagogy and Education, 20(3), 301-316. doi: https://doi.org/10.1080/1475939X.2011.610931

Loveless, A., \& Williamson, B. (2013). Learning identities in a digital age: Rethinking creativity, education and technology. New York, NY: Routledge.

Marhuenda-Fluixá, F. (2012). La Formación Profesional. Madrid: Síntesis.

Marhuenda-Fluixá, F. \& Ros-Garrido, A. (2015). What sense can we make of the possibility of vocational didactics? An approach from the Spanish school-based system complemented by non-formal vocational training. International journal for research in vocational education and training, 2(3), 170-181. doi: https://doi.org/10.13152/IJRVET.2.3.3

Marhuenda-Fluixá, F., Chisvert-Tarazona, M. J., Palomares-Montero, D., y Vila, J. (2017). Con d de dual: investigación sobre la implantación del sistema dual en la formación profesional en España. Educar, 53(2), 285-307. doi: https://doi.org/10.5565/rev/educar.821

Martínez, M. (2006). La investigación cualitativa, Revista IIPSI, 9(1), 123-146. doi: http://dx.doi.org/10.15381/rinvp.v9i1.4033

MECD (2013). Marco Común de Competencia Digital Docente. V. 2.0. Disponible en http://educalab.es/documents/10180/12809/MarcoComunCompeDigiDoceV2.pdf/e8 766a69-d9ba-43f2-afe9-f526f0b34859

Mishra, P. y Koehler, M. (2006). Technological pedagogical content knowledge: A Framework for Teacher knowledge. Teachers College Record, 108(6), 1017-1054. doi: https://doi.org/10.1111/j.1467-9620.2006.00684.x

Montanero, M.M. (2019). Métodos pedagógicos emergentes para un nuevo siglo ¿Qué hay realmente de innovación? Teoría de la Educación. Revista Interuniversitaria, 31(1), 5-34. doi: http://dx.doi.org/10.14201/teri.19758

Morris, S. (2013). Decoding Digital Pedagogy, pt. 1: Beyond the LMS. Hybrid Pedagogy, https://hybridpedagogy.org/decoding-digital-pedagogy-pt-1-beyond-the-lms/

Organización para la Cooperación y Desarrollo Económico (OCDE) (2011). Preparándose para trabajar. Barcelona: Fundación Barcelona FP. doi: https://doi.org/10.1787/9789264118478-es

Organization for Economic Cooperation and Development (2019). TALIS 2018 Results (Volume I), Teachers and school leaders as lifelong learners. Paris: OECD. doi: https://doi.org/10.1787/23129638

Peters, M. A., Liu, T.C., \& Ondercin, D.J. (2012). The Pedagogy of the Open Society. doi: https://doi.org/10.1007/978-94-6091-967-1

Prensky, M. (2001). Digital natives, digital immigrants part 1. On the horizon, 9(5), 1-6. doi: https://doi.org/10.1108/10748120110424816

Redecker, C. (2017). European framework for the digital competence of educators: DigCompEdu (No. JRC107466). Joint Research Centre. doi: https://doi.org/10.2760/159770

Ros-Garrido, A., \& Marhuenda-Fluixá, F. (2019). The Education of VET Teachers and Trainers. In F. Marhuenda-Fluixá (ed.). The School-Based Vocational Education and Training System in Spain (pp. 87-103). Springer, Singapore. doi: https://doi.org/10.1007/978-981-13-8475-2

Salas-Ruiz, J. y Sánchez-Rivas, E. (2019). Incorporación de aplicaciones informáticas en la Formación Profesional. Un estudio de caso. En E. Sánchez, J. Ruiz y E. Sánchez, Innovación y tecnología en contextos educativos ( $\mathrm{pp}$. 751-759). Málaga: UMA editorial. 
Salinas, J., De Benito, B., y Lizana, A. (2014). Competencias docentes para los nuevos escenarios de aprendizaje. Revista Interuniversitaria de Formación del Profesorado, $78(1), 145-163$.

Saran, S.; Chapman, T. y Sharma, M. (2018). A New Social Contract for the Digital Age. Disponible en: https://t20argentina.org/wp-content/uploads/2018/05/A-New-SocialContract-for-the-Digital-Age-Vision-Brief-ORF-4-10-18-TC_Edits-3.pdf

Stödberg, U. (2012). A research review of e-assessment. Assessment y Evaluation in Higher Education, 37(5), 591-604. doi: https://doi.org/10.1080/02602938.2011.557496

Strauss, A. L. y Corbin, J. (2002). Bases de la investigación cualitativa: técnicas y procedimientos para desarrollar la teoría fundada ( $l^{a}$ ed.). Medellín: Editorial Universidad de Antioquia.

Suárez-Guerrero, C. (2014) Pedagogía red, Cuadernos de pedagogía, 449, 76-80. Disponible en: http://bit.ly/1nfogNf

Suárez-Guerrero, C., Lloret-Catalá, C. \& Mengual, S. (2016). Teachers' Perceptions of the Digital Transformation of the Classroom through the Use of Tablets: A Study in Spain. Comunicar, 49, 81-89. doi: https://doi.org/10.3916/C49-2016-08

Suárez-Guerrero, C; Lizandra, J. y Ros, A. (2020). Análisis pedagógico de la competencia digital docente en la educación técnico profesional, Libro de ponencias, XXII Congreso Internacional Tecnología e innovación para la diversidad de los aprendizajes EDUTEC 2019, pp. 948-962. Lima: PUCP.

Szymielewicz, K. (2019). Your digital identity has three layers, and you can only protect one of them. Disponible en: https://qz.com/1525661/your-digital-identity-has-threelayers-and-you-can-only-protect-one-of-them/

Turner, D. (2017). The learning wheel: A model of digital pedagogy. Social Work Education, 36(8), 959-960. DOI: https://doi.org/10.1080/02615479.2017.1331529

UNESCO (2008). ICT competency standard for teachers. Disponible en: https://unesdoc.unesco.org/ark:/48223/pf0000156210

UNESCO (2011). ICT Competency Framework for Teachers Version 2.0. UNESCO. Disponible en: https://iite.unesco.org/pics/publications/en/files/3214694.pdf

Valtonen, T., Pontinen, S., Kukkonen, J., Dillon, P., Väisänen, P., \& Hacklin, S. (2011). Confronting the technological pedagogical knowledge of Finnish Net Generation student teachers. Technology, Pedagogy and Education, 20(1), 3-18. doi: https://doi.org/10.1080/1475939X.2010.534867

Valverde, J. (2010). El movimiento de "Educación Abierta" y la "Universidad Expandida", Tendencias Pedagógicas, 16, 157-180. Disponible en. https://revistas.uam.es/tendenciaspedagogicas/article/view/1948/2059

Van Acker, F., Van Buuren, H., Kreijns, K., \& Vermeulen, M. (2013). Why teachers use digital learning materials: The role of self-efficacy, subjective norm and attitude. Education and Information Technologies, 18(3), 495-514. doi: https://doi.org/10.1007/s10639-011-9181-9

Veletsianos, G. (2011). Designing opportunities for transformation with emerging technologies. Educational Technology, 41-46. doi: http://dx.doi.org/10.25316/IR-44

Vuorikari, R., Punie, Y., Carretero S., \& Van den Brande, G. (2016). DigComp 2.0: The Digital Competence Framework for Citizens. Update Phase 1: The Conceptual Reference Model. Luxembourg: Publication Office of the European Union. doi: https://doi.org/10.2791/11517

Aproximación a la competencia digital docente en la formación profesional. Suárez-Guerrero, Ros-Garrido y Lizandra. 
White, D. S., \& Le Cornu, A. (2011). Visitors and Residents: A new typology for online engagement. First Monday, 16(9). doi: https://doi.org/10.5210/fm.v16i9.3171

Wiley, D. (2013). What is Open Pedagogy? Disponible en: https://opencontent.org/blog/archives/2975

Williams, P. (2017). Assessing collaborative learning: big data, analytics and university futures. Assessment y Evaluation in Higher Education, 42(6), 978-989. doi: https://doi.org/10.1080/02602938.2016.1216084

Williamson, B (2018). Big Data en Educación. El futuro digital del aprendizaje, la política y la práctica. Madrid: Morata.

World Economic Forum. (2018). The Global Competitiveness Report 2017-2018. Disponible en: https://www.weforum.org/reports/the-global-competitiveness-report2017-2018 